\title{
Deprived children or deprived neighbourhoods? A public health approach to the investigation of links between deprivation and injury risk with specific reference to child road safety in Devon County, UK
}

\author{
Paul Hewson*
}

Address: Environment Directorate, Devon County Council, County Hall, Topsham Road, Exeter, UK

Email: Paul Hewson* - phewson@devon.gov.uk

* Corresponding author

Published: 10 May 2004

BMC Public Health 2004, 4: 15
Received: 07 November 2003

Accepted: 10 May 2004

This article is available from: http://www.biomedcentral.com/I47/-2458/4//5

(c) 2004 Hewson; licensee BioMed Central Ltd. This is an Open Access article: verbatim copying and redistribution of this article are permitted in all media for any purpose, provided this notice is preserved along with the article's original URL.

\begin{abstract}
Background: Worldwide, injuries from road traffic collisions are a rapidly growing problem in terms of morbidity and mortality. The UK has amongst the worst records in Europe with regard to child pedestrian safety. A traditional view holds that resources should be directed towards training child pedestrians. In order to reduce socio-economic differentials in child pedestrian casualty rates it is suggested that these should be directed at deprived children. This paper seeks to question whether analysis of extant routinely collected data supports this view.

Methods: Routine administrative data on road collisions has been used. A deprivation measure has been assigned to the location where a collision was reported, and the home postcode of the casualty. Aggregate data was analysed using a number of epidemiological models, concentrating on the Generalised Linear Mixed Model.

Results: This study confirms evidence suggesting a link between increasing deprivation and increasing casualty involvement of child pedestrians. However, suggestions are made that it may be necessary to control for the urban nature of an area where collisions occur. More importantly, the question is raised as to whether the casualty rate is more closely associated with deprivation measures of the ward in which the collision occurred than with the deprivation measures of the home address of the child.

Conclusion: Conclusions have to be drawn with great caution. Limitations in the utility of the officially collected data are apparent, but the implication is that the deprivation measures of the area around the collision is a more important determinant of socio-economic differentials in casualty rates than the deprivation measures of the casualties' home location. Whilst this result must be treated with caution, if confirmed by individual level case-controlled studies this would have a strong implication for the most appropriate interventions.
\end{abstract}

\section{Background}

A recent editorial in the British Medical Journal suggested that "by 2020 road traffic crashes will have moved from ninth to third place in the world ranking of the burden of disease and will be second place in developing countries" [1]. This editorial also highlighted inequities in road 
traffic injury. Associations between deprivation and health generally are well studied; the actual mechanisms underlying this association are not always so well characterised. Of more concern is evidence that in terms of differential injury rates, the gaps are widening [2]. Hospital based studies confirm the association between deprivation and a wide range of injury types, including pedestrian injury [3]. In the UK it appears that children from lower socio-economic status backgrounds may be up to five times as likely to be injured as pedestrians than children from higher socio-economic status backgrounds [4]. Much effort in terms of social marketing and education has been directed on the assumption that there are behavioural factors associated with children from lower socioeconomic status backgrounds that explains the exaggerated casualty risk. Systematic review highlights a small number of studies that suggest that child behaviour can be modified by educational intervention [5], but these have not proven an associated reduction in casualty involvement. It has been argued that however well educated, children remain children and make mistakes [6]. Given evidence that drivers rarely alter their behaviour in the presence of children [7], the most important solution to the child pedestrian health problem would be environmental modification that makes the environment more forgiving of childhood "errors". Certainly, systematic reviews here do point to their effectiveness in reducing casualty involvement [8]. It has been lamented that public health methodology is under-used within the road safety arena [9] and so it is therefore of some interest to consider what can be gleaned from epidemiological analysis of the routinely collected data on road collisions.

In the UK, the Institute of Public Policy Research (IPPR) has recently released a major study considering an econometric analysis of the association between deprivation and child casualty involvement [10]. This study used an official deprivation measure commissioned by the Department for the Environment, Transport and the Regions (DETR) from Oxford University [11]. Overall, this index aimed to capture multiple deprivation, for example combining domains relating to income, access, health and housing measures. However, in relation to Child Deprivation, the index was solely income based, and measured children in households in receipt of a number of state benefits, including income support, income based job-seekers allowance, family credit and disability working allowance. The IPPR study found a "strong" correlation between child pedestrian casualty rates and the deprivation index, but did note that there was a great deal of variation between wards with similar deprivation scores. Covariates were also included for road lengths, junction counts, employment conditions and weather conditions. In contrast with earlier work on pedestrian exposure [12] the suggestion was made that the greater the number of minor roads, the greater the number of pedestrian casualties and the authors concluded that deprivation effects were not related to the speed, volume and type of traffic present in deprived wards. This is significant because these are the very environmental features that have been shown in systematic reviews to be amenable to modification leading to an effective reduction in injury [8]. One difficulty that remains with this type of analysis that it is extremely susceptible to the ecological fallacy [13]. This is a pity as recent work specifically concerning the association between deprivation and health has highlighted the potential to use latent variable techniques, for example to construct an age adjusted deprivation index that explained most of the variation in rates of illness across a study region [14]. Another difficulty with the IPPR work is that it remains unclear whether any apparent association between deprivation indices and child pedestrian casualty rates are due to the child or the area where the collision occurred. If the association is with the child, their socioeconomic status can be classified by the index pertaining to the location of their home address. If the area where the collision occurred is more important it can be measured in terms of the index pertaining to the location of the collision. It is of great interest to extend the previous econometric modelling to see whether the routinely available data can provide any evidence in relation to this question.

The UK Government has commissioned research which reviewed both socio-economic factors and environmental factors and identified a number of environmental risk factors, such as pre-1916 housing estates (long straight roads), number of three and four-way junctions and absence of traffic calming [15]. This work suggested that a variety of interventions aimed at traffic calming, reducing traffic flow in residential areas as well as improved crossing facilities in residential areas could be effective in reducing casualty rates. More recent research has been commissioned comparing the UK with some of its European neighbours [16]. This has suggested that a number of environmental factors, such as the need to cross more heavily trafficked roads and greater reliance on unmarked crossings, may in part explain the relatively poor history of child pedestrian casualty rates in the UK.

This paper therefore sets out detailed modelling work using the routinely available official data source on road collisions in the United Kingdom. One potential area for introduction of the ecological fallacy has been examined; by controlling for the urbanicity of the wards over which the data is aggregated. The aim of the study is to see whether epidemiological assessment of this data supports the view of a link between child deprivation and elevated child pedestrian casualty rates. In particular, it is of interest to determine whether the strength of evidence for this 
association is between the ward containing the recorded home postcode of the child or the ward containing the location of the collision.

\section{Methods}

\section{Location and geographical context of the study}

Devon County is located in the Southwest peninsula of England, UK. Devon County Council came into being following the Local Government Act of 1888 and its current structure reflects the latest round of local government reorganisation, which in 1998 removed the two largest urban areas (Plymouth and Torbay) from its governance. The major centre of population remaining within Devon County is the cathedral city of Exeter (population $110,000)$. There are no medium size towns with populations between 50,000 and 100,000 but there are a number of significant urban settlements such as Barnstaple (population 20,800), Newton Abbot (population 23,600) and Exmouth (population 32,400). In terms of its Highways Authority function, the council is responsible for maintaining $12,658 \mathrm{~km}$ of road, more than any other Highways Authority in England. In addition to roads under the Council's control, the Highways Agency for England maintains $38 \mathrm{~km}$ of Motorway and $273 \mathrm{~km}$ of Trunk road within Devon's boundaries.

The population in Devon is currently estimated as 706,000 with a child population of 120,000 . The administrative geography of Devon is further broken into 232 wards with an average of 530 children per ward and 2600 adults. The 1998 ward boundaries have been used for this study as the data regarding the child deprivation index was made available by the Office for National Statistics based in the 1998 boundaries. Definitions of Rural and Urban follow the recommendation of the Office of the Deputy Prime Minister in the United Kingdom [17].

Excluding the seven metropolitan areas of England, namely Greater London, Greater Manchester, West Yorkshire, South Yorkshire, Merseyside, Tyne and Wear and West Midlands, Devon is average in terms of child pedestrian casualty count. The average number of child pedestrians reported killed or seriously injured in any of the non-metropolitan Highways Authority areas in 2002 was 16 , with a further 65 reported as having incurred slight injuries [18]. In Devon there were 18 reported killed and seriously injured child pedestrians with a further 94 reported slight pedestrian injuries in 2002. Whilst Devon contains a large and very sparse rural element, there are clearly a number of urban areas within the County, and the casualty figures are not atypical for Highways Authorities in England. Taking the Shire County of Devon as as a whole, Devon is the tenth out of 38 English Counties in terms of its rural nature, having a total of $22 \%$ of its population resident in rural areas [19].

\section{Data}

For the purposes of this study, a Child is defined as being in the age range $0-15$ years.

The "definitive" source of information on road casualties within the UK comes from data collected by the police on a form referred to as "Stats 19", hence the data is subsequently also referred to as "Stats 19 data". These data were first collected in the UK in 1919, but a formalised regime for data collection was established in 1949 [20]. Arrangements are made by the local processing authority (which may be the Police, the Local Authorities or a subcontractor depending on local arrangements) to return this data to the Department for Transport (DfT). There are specified national requirements for data collection, but additional information may be collected locally and some of this additional local information is used here in determining the ward of residence of the casualty. It has long been recognised that there are large problems associated with the capture of information within this system [21]. Of particular relevance to this study, a meta-analysis of 25 studies has suggested that collisions involving vulnerable road users and young road users are most likely to be underreported [22]. Attempts to match the Police reports of collisions with in-patient records in the UK have suggested that generally, around $60 \%$ of slight collisions are both reported to and recorded by the Police $[23,24]$. It is therefore an unfortunate feature when using the "official" data source on child pedestrian casualties that is known to be the most unreliable. It was suggested two decades ago that Police reporting of road casualties be supplemented by the involvement of Health Professionals, and also that the scale of road injury generally should be validated by survey [25]. However, these suggestions are yet to be followed up. There is clear potential to study this issue from a Hospital perspective, however by definition minor injuries will be excluded from in-patient data. The Police data therefore remains an important source of information on road casualty involvement. As it is the benchmark dataset with respect to road casualties in the UK, it is the data source considered here.

Previous work has used the casualty postcode to infer a number of demographic factors relating to the casualty [26]. Unfortunately this element of the data is not collected centrally and so it is necessary to concentrate on a local study. Information on collisions in which 980 children were injured as pedestrians within Devon between January 1996 and December 2002 has been extracted for further analysis. Of these, valid postcode information from within Devon was available for 372 casualties. These data were aggregated in two ways. Firstly, counts of the number of reported child casualties by the ward in which the collision occurred were calculated. A scatter plot of the casualty rate relative to the child population of the ward 


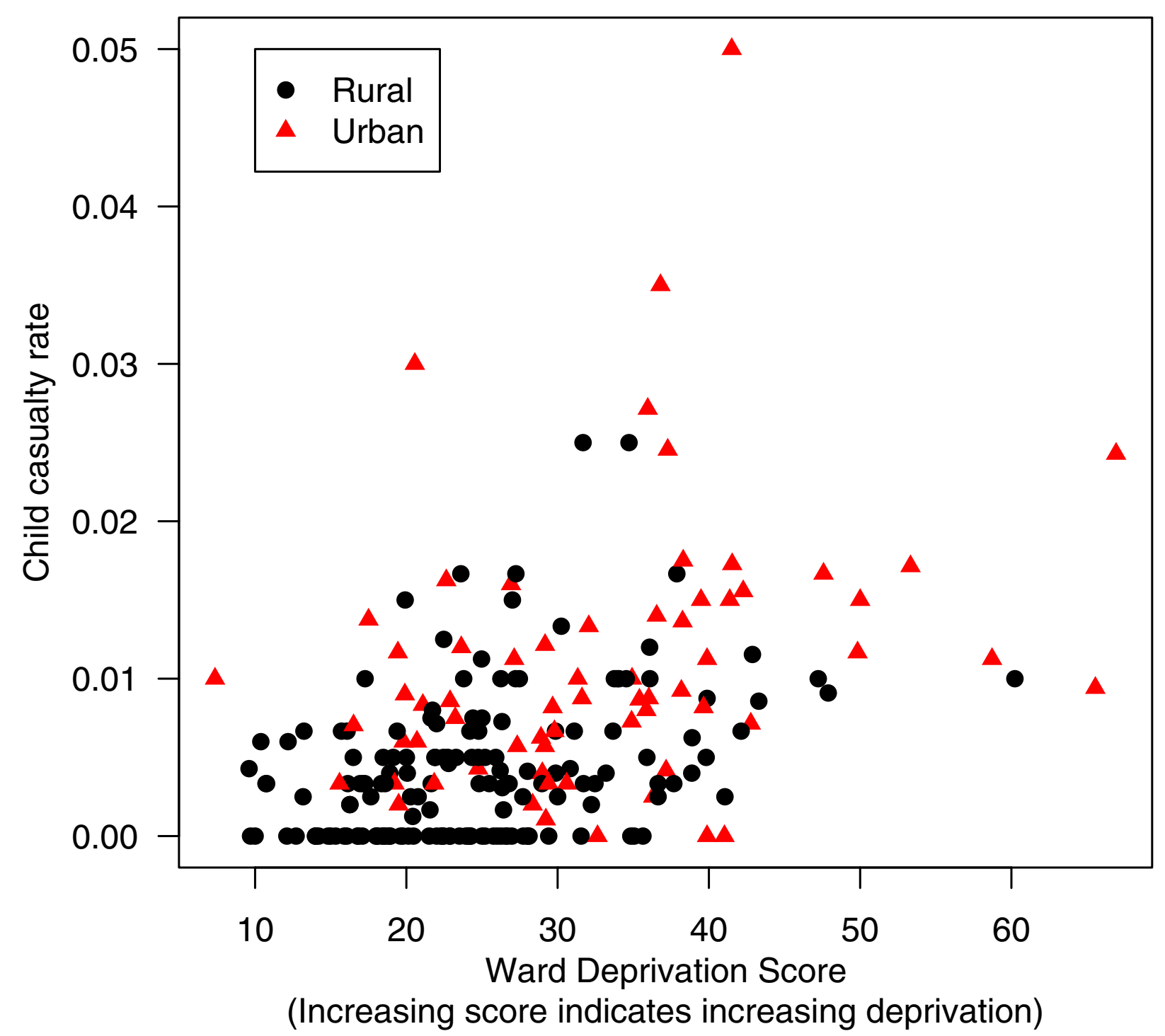

Figure I

Child casualty rates by ward of collision. Child Casualty rates plotted against ward deprivation score (aggregated by the ward in which thecollision occurred, casualties reported injured in Devon 1996-2002)

for these data is given in Figure 1, and a frequency distribution depicting the number of casualties reported injured in collisions which occurred in rural and urban wards is given in Table 1. Secondly, where casualty postcode information was available, counts of reported casu- alties by the home of the casualty were calculated. The casualty ward was taken as the ward that coincided with the centre of the postcode sector given as the casualty postcode. Similarly, a scatter plot of the casualty rate relative to the child population of the ward for these data is 
Table I: Children reported injured in collisions in Devon by ward of collision. Frequency distribution for the number of child pedestrian casualties per ward in Devon reported between 1996 and 2002, aggregated by the ward in which the collision occurred.

\begin{tabular}{|c|c|c|c|c|c|c|c|c|c|c|c|c|c|c|}
\hline $\begin{array}{l}\text { Number of casualties } \\
\text { per ward }\end{array}$ & 0 & 1 & 2 & 3 & 4 & 5 & 6 & 7 & 8 & 9 & 10 & $11-15$ & $16-20$ & $21-30$ \\
\hline Number of Rural wards & 56 & 38 & 23 & 11 & 7 & 7 & 8 & 4 & 3 & I & 2 & 5 & 0 & 0 \\
\hline Number of Urban wards & 3 & 2 & 7 & 6 & 2 & 4 & 4 & 5 & 4 & 7 & 1 & 10 & 8 & 4 \\
\hline
\end{tabular}

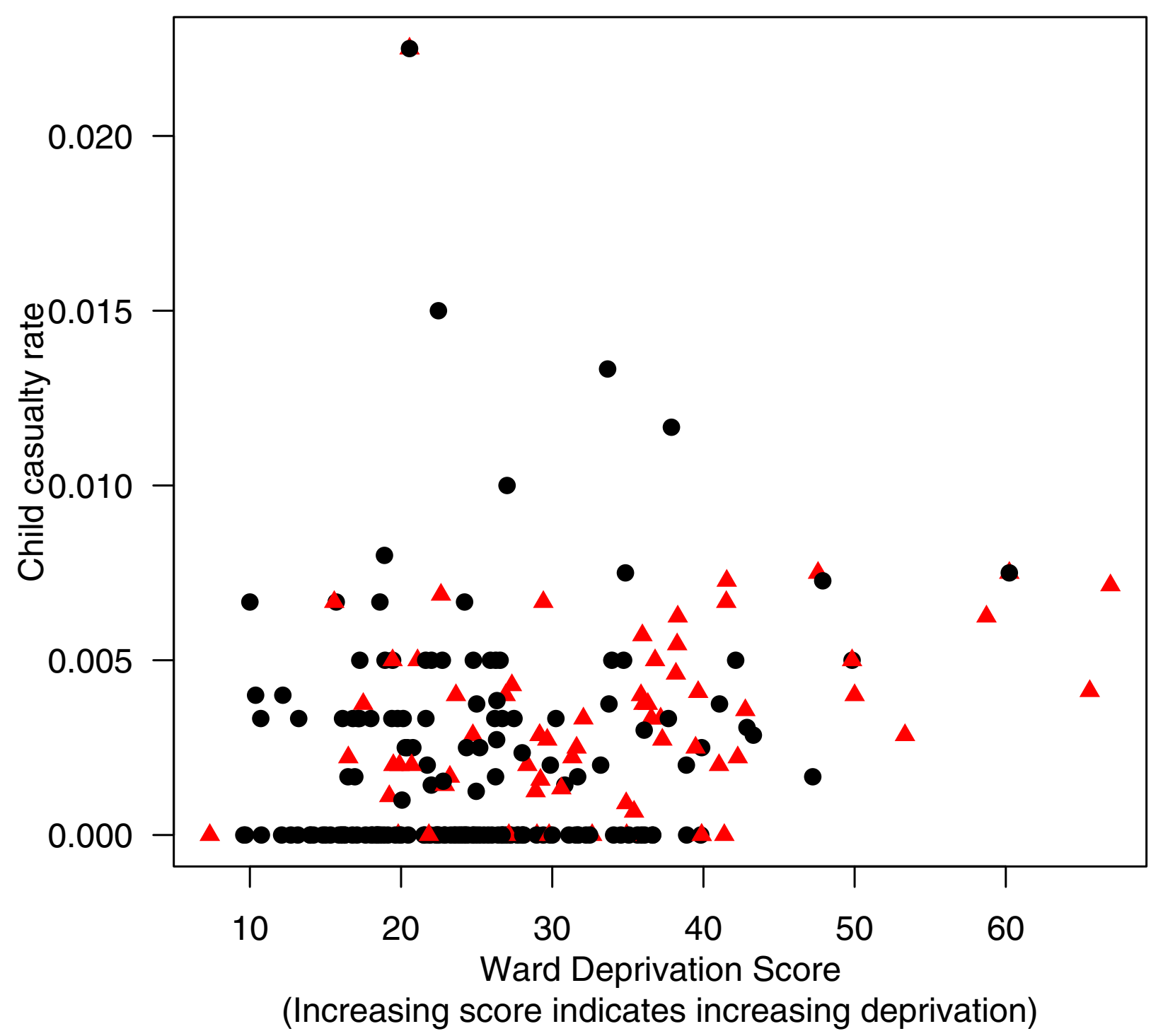

Figure 2

Child casualty rates by ward of casualty residence. Child Casualty rates plotted against ward deprivation score (aggregated by the ward of casualty residence, casualties reported injured in Devon 1996-2002) 
Table 2: Children reported injured in collisions in Devon by ward of residence. Frequency distribution for the number of child pedestrian casualties per ward in Devon reported between 1996 and 2002, aggregated by the ward in which the casualty postcode was located.

\begin{tabular}{|c|c|c|c|c|c|c|c|c|c|c|c|c|c|c|}
\hline $\begin{array}{l}\text { Number of casualties } \\
\text { per ward }\end{array}$ & 0 & I & 2 & 3 & 4 & 5 & 6 & 7 & 8 & 9 & 10 & $|I-| 5$ & $16-20$ & $21-30$ \\
\hline Number of Rural wards & 91 & 35 & 15 & II & 7 & I & I & I & I & 2 & 0 & 0 & 0 & 0 \\
\hline Number of Urban wards & II & 9 & 15 & 10 & 7 & 5 & 3 & I & I & 4 & 0 & 1 & 0 & 0 \\
\hline
\end{tabular}

Table 3: Basic GLM fitted to data aggregated by ward of collision. Results from fitting a generalised linear model with Poisson errors to the ward casualty rates calculated for the ward in which the collision occurred.

\begin{tabular}{|c|c|c|c|c|}
\hline & Estimate & Standard Error & $\mathrm{z}$ value & $\operatorname{Pr}(\geq|z|)$ \\
\hline Intercept & -5.839 & 0.090 & -64.65 & 0.00 \\
\hline Deprivation & 0.021 & 0.002 & 8.09 & 0.00 \\
\hline Urbanicity & 0.512 & 0.069 & 7.39 & 0.00 \\
\hline
\end{tabular}

presented in Figure 2, and a frequency distribution giving the number of reported casualties resident by rural and urban wards is given in Table 2. Care was taken to investigate whether there was any evidence for bias in the missing postcodes. No significant bias was apparent in terms of the ward location of the collision and missing casualty postcodes. It is therefore not felt that this is likely to influence the results of this particular study. However, given that considerable under-reporting and under-recording of any child pedestrian casualty can be assumed, and that just over a third of these have valid postcodes it is clear that the valid data represents a small fraction of the children who are likely to have been injured as pedestrians.

\section{Models}

Modelling the casualty rate

The casualty rate is an expression of the number of reported casualties considered in relation to the relevant population in a given area. This can be most appropriately modelled in an analogous manner to the Standardised Morbidity Ratio (SMR). The count of casualties can be assumed to be capable of representation by the Poisson family (or some extension of it). In this context, the expected morbidity count of the SMR can be replaced by the "Population at risk", here the nominal population of children aged $0-15$ in each ward.

The Generalised Linear Model (GLM): basic Poisson model

The basic model that would be used to investigate this data in an epidemiological setting is the Poisson model with a population offset [27].

$Y_{i} \sim \operatorname{Poisson}\left(o_{i} \lambda_{i}\right)$
Where $Y_{i}$ is the casualty count in a given ward, $o_{i}$ is the relevant population count in a given ward and $\lambda_{i}$ is the casualty rate. A generalised linear model can be applied which allows us to model the casualty rate $\lambda_{i}$ as a function of a linear predictor whereby:

$\log \left(\lambda_{i}\right)=\mu_{i}$

and $\mu_{i}$ can be modelled by a linear function of an intercept $(\alpha)$ and covariates.

$\mu_{i}=\alpha+\beta_{i} x_{i}$

However, in both epidemiology and road safety it is now commonplace to use much more elaborate models that can realistically represent the data more faithfully. The negative binomial was one of the earlier ones to be used in road safety [28], but generalised additive models [29] and h-likelihood models [30] have been applied. More recently though, comparisons of the negative binomial and Poisson log-normal model gave substantively similar results [31], therefore it is reasonable to consider the following generalised linear mixed model (GLMM):

The Generalised Linear Mixed Model (GLMM): Poisson model with log-normal random effects

This is based on the basic Poisson model above:

$Y_{i} \sim \operatorname{Poisson}\left(o_{i} \lambda_{i}\right)$

But now, an additive random effect is included, modelled from a zero-mean normal distribution (denoted by $v_{i}$ within the casualty rate parameter $\lambda_{i}$ ) such that: 
Table 4: Basic GLM fitted to data aggregated by casualty home ward. Results from fitting a generalised linear model with Poisson errors to the ward casualty rates calculated for the ward in which the casualty was resident

\begin{tabular}{|c|c|c|c|c|}
\hline & Estimate & Standard Error & $\mathrm{z}$ value & $\operatorname{Pr}(\geq|z|)$ \\
\hline Intercept & -6.645 & 0.144 & -46.192 & 0.00 \\
\hline Deprivation & 0.021 & 0.004 & 5.050 & 0.00 \\
\hline Urbanicity & 0.202 & 0.110 & 1.833 & 0.07 \\
\hline
\end{tabular}

Table 5: GLMM fitted to data aggregated by ward of collision. Results from fitting a generalised linear mixed model with Poisson errors and log-normal random effects to the ward casualty rates calculated for the ward in which the collision occurred.

\begin{tabular}{|c|c|c|c|c|}
\hline & Estimate & Standard Error & $\mathrm{t}$ value & $\operatorname{Pr}(\geq|\mathrm{t}|)$ \\
\hline Intercept & -0.024 & 0.172 & -0.140 & 0.89 \\
\hline Deprivation & 0.030 & 0.005 & 5.986 & 0.00 \\
\hline Urbanicity & 1.089 & 0.138 & 7.906 & 0.00 \\
\hline
\end{tabular}

Table 6: GLMM fitted to data aggregated by casualty home ward. Results from fitting a generalised linear model with Poisson errors and log-normal random effects to the ward casualty rates calculated for the ward in which the casualty was resident.

\begin{tabular}{|c|c|c|c|c|}
\hline & Estimate & Standard Error & $\mathrm{z}$ value & $\operatorname{Pr}(\geq|t|)$ \\
\hline Intercept & -0.813 & 0.217 & -3.753 & 0.00 \\
\hline Deprivation & 0.032 & 0.007 & 4.862 & 0.00 \\
\hline Urbanicity & 0.765 & 0.173 & 4.414 & 0.00 \\
\hline
\end{tabular}

$\log \left(\lambda_{i}\right)=\mu_{i}+v_{i}$

As above, $\mu_{i}$ can be modelled as a linear function Both models can be applied using the R software $[32,33]$. There are a wide range of methods for fitting generalised linear mixed models, here penalised quasi-likelihood was used [34]. Random effects can be considered as having a number of roles in such a model. For example they provide one way of accounting for "over-dispersion" in the data, i.e. there is more variability in the real-world data than suggested by simple statistical theory. Random effects can also be considered as unmeasured latent variables, which account for unmeasured covariates. This is an extremely important consideration in this context, as we can be sure that the proxy variables measured do not represent all the systematic differences between the road environment in the various wards.

In summary, two scenarios have been investigated, one whereby the casualty count is aggregated by the ward in which the collision was reported, and one where the casualty count is aggregated by the ward in which the casualty postcode occurs.

\section{Results}

A number of standard graphical assessments were made of model fit, such as Quartile-Quartile plots, Cooks Distance Plot, Scale-Location Plots and other residual scatter plots to check for the possible influence of outliers and the validity of the residuals obtained. These highlight problems with the simple Poisson models, but strongly suggest that over-dispersion problems are adequately dealt with by the incorporation of the random effects.

\section{GLM: basic Poisson model}

Tables 3 and 4 demonstrate the results from fitting simple Poisson models to the child pedestrian data fitted to the child pedestrian data aggregated by the ward in which the collision occurred and the ward in which the casualty was resident respectively. In both cases, the estimate of the deprivation covariate is positive $(0.021$ and 0.021 respectively with confidence intervals of $0.017,0.025$ and 0.013 , 0.029 respectively), indicating that according to these 
models the casualty rate increases as the deprivation measure increases. When the data has been aggregated by the ward in which the collision occurred, there is evidence that urban wards have a higher casualty rate than rural ones. This cannot be stated with the same certainty when the data is aggregated by the ward in which the casualty was resident. The $95 \%$ confidence interval on the estimate for the urbanicity parameter includes zero, which allows for the possibility that it had no effect.

However, the graphical diagnostics for these models demonstrate some problems, particular in terms of influential points. The Residual deviance is (respectively) 545.59 and 335.85 on 229 degrees of freedom, which provides evidence for over-dispersion, and justifies the use of a more complex model.

\section{GLMM: Poisson model with normal random effects}

Tables 5 and 6 give the parameter estimates for the GLMM (Poisson model with normal random effects) fitted to the child pedestrian data aggregated by the ward in which the collision occurred and the ward in which the casualty was resident respectively. The Akaike Information Criteria for these models is 697.2 and 791.9 respectively (the negative log-likelihoods are 343.6 and 390.9 respectively). There is evidence in both cases of a positive association between the deprivation indicator and the casualty rate, whether aggregated by the ward in which the collision occurred or the ward of residence of the casualty. Also, there is strong evidence for the inclusion of an urbanicity component in the model. When the generalised linear mixed model is used, the estimate for the deprivation parameter is 0.030 (confidence intervals 0.020, 0.030) when considering the results by collision location whereas this parameter is estimated at 0.021 (confidence intervals $0.017,0.025$ ) in the simple model. Equally, the estimate for the deprivation parameter from the generalised linear mixed model applied to the data aggregated by home postcode is 0.032 (confidence intervals $0.018,0.046$ ) compared with 0.021 (confidence intervals $0.013,0.029$ ) for the results without random effects. It is noteworthy that the confidence intervals from the two models overlap, but that they are much wider when using the GLMM, which indicates greater uncertainty about the degree of association.

Inference is however altered in the case of the urbanicity parameter. With the data aggregated by the collision location the estimate of the role of urbanicity has increased from 0.512 (confidence intervals $0.374,0.650$ ) to 1.089 (confidence intervals $0.813,1.365$ ). However, when the aggregation is carried out by casualties home, the estimate is changed from one where the confidence intervals include zero 0.202 (confidence intervals - $0.018,0.422$ ) to one that is clearly positive 0.765 (confidence intervals $0.419,1.111$ ). Including random effects in the model

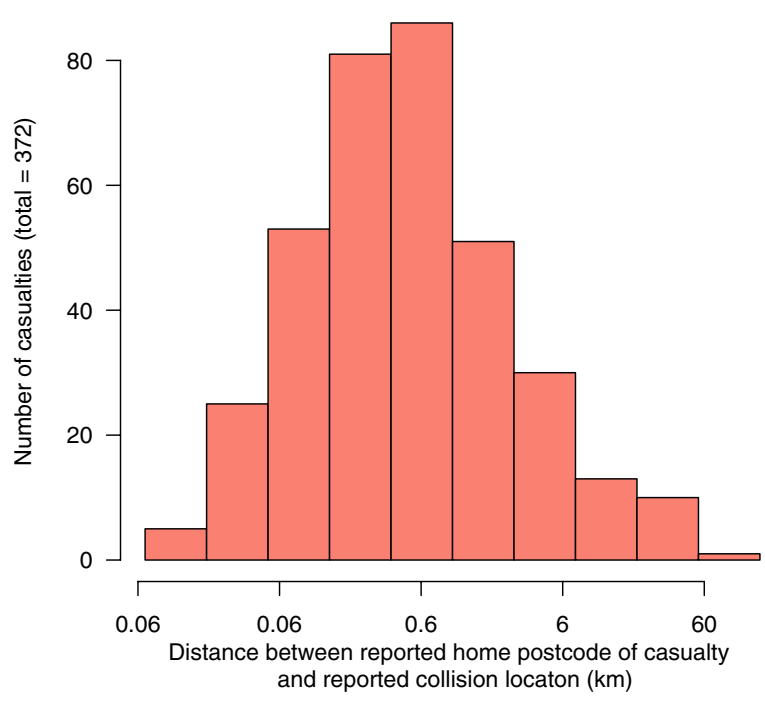

\section{Figure 3}

Distance from home. Distance of child pedestrian casualties from reported home (reported injured in collisions in Devon between 1996 and 2002).

increases the uncertainty about the association between the casualty rate and the deprivation score, but decreases the uncertainty of the association between casualty rate and urbanicity.

Exploration of the data confirms that most child pedestrian casualties occur near the home postcode of the child. This is depicted (on a log scale) in Figure 3. This therefore means that usually, the deprivation score for the area of where the collision occurred and the deprivation score associated with the casualty are actually the same. A small difference in model fit is therefore quite significant in terms of the small number of children who were injured away from their area of residence.

\section{Discussion}

This study has set out to determine what can be gleaned about the relationship between deprivation and exaggerated child pedestrian casualty risk. At the outset of this study, many caveats on the validity of the official data source are clearly signalled in the literature and are consistent with the findings here. However, this does not obviate the need to make the best statistical inference that is possible given an expensively collected, official benchmark dataset. 
Controlling for urbanicity has been used to illustrate the potential for using latent variables to correct for the ecological fallacy. The association between deprivation and child pedestrian casualty risk is much lower when this is controlled for, and highlights the potential for masking when additional variables were included. There is an obvious mechanism for the importance of urbanicity as a covariate. Urban wards have very different traffic patterns to rural ones, and also tend to demonstrate a higher level of deprivation as measured by the child deprivation index used here. As a result, at a meta-aggregate level the situation is one where urban wards tend to have both higher deprivation measures and higher child pedestrian casualty rates. One possible answer to the ecological fallacy with respect to deprivation may be to use the Council Tax valuation band [35], which could be applied at the individual level, and could be collected in a way that guarantees more protection of the individuals privacy.

Clearly great caution has to be taken with the results of this study. Part of the reason for this caution is because, if confirmed, the implications can be quite profound. With confirmation, the ultimate implication of this work could be to suggest that the environment where the collision took place is of some importance in terms of explaining the variable casualty risk. The consequence of this suggestion is clearly that environmental modifications would be more important than attempting to modify the behaviour of children. Interestingly, evidence from the literature suggests that environmental modifications can alter casualty rates [8], whilst evidence is still awaited in respect of the casualty reduction potential of educational interventions [5].

However, appropriate confirmation of the findings of this study is necessary. There is an alternative view to improving the quality and coverage of routine data. Given that extensive statistical analysis of a specific, and high profile question fails to produce a conclusive answer the best method for answering this question appears to be by a hospital based case-controlled study. Perhaps some of the resources devoted to attempting to collect a definitive data source would be better directed towards focussed studies of relevant issues. For some reason, there is a dearth of such studies carried out in the UK context. Such work, although not specifically addressing the question of child deprivation has been carried out in Australia [36] and emphasised the importance of a number of environmental factors (traffic flow corrected speed and kerb parking) as being relevant in explaining casualty risk.

\section{Conclusions}

In drawing a conclusion from this study it is essential to highlight the obvious potential for over-interpreting the data. Certainly, there is some evidence at the aggregate level for an association between deprivation and increased child pedestrian casualty risk. However, extensive analysis, using the official data source still leaves some ambiguity as to whether this relationship is more strongly associated with the deprivation measures related to the home postcode of the casualty or with the location of the collision. In the light of this study and other literature, it can be suggested that it is not possible to rule out the role for environmental factors in terms of explaining this increased risk.

The limitations of the official data source are highlighted by this study. Two decades ago, suggestions were made that initial reports by the police should be completed by medical personnel, initially to gain a more accurate picture of severity of injury [23]. Extensive additional data collection occurs in respect of injury, such as paramedic staff in ambulances, accident and emergency staff in hospitals as well as in-patient systems. It seems reasonable to aspire to capture much better depth and quality of data in respect of road traffic casualties. However, cost-effectiveness considerations may make a better argument in favour of focussed studies, such as case-control studies to elucidate the risk factors at an individual level than in expanding the routine data collection to obtain all the data that may be required to a suitable standard.

\section{Competing interests}

None declared.

\section{Acknowledgements}

Devon and Cornwall Constabulary supplied the collision data.

\section{References}

I. Roberts I, Mohan D, Abbasi K: War on the roads. Brit Med J 2002, 324: I1 07-1108.

2. Roberts I, Power C: Does the decline in child injury mortality vary by social class? A comparison of class specific mortality in I98I and I99 I. Brit Med J 1997, 3 I 3:784-786.

3. Lyons R, Jones S, Deacon T, Heaven M: Socio-economic variation in injury in children and older people: a population based study. Injury Prevention 2003, 9:33-37.

4. Thomson JA, Tolmie A, Mamoon T: Road accident involvement of children from ethnic minorities: a literature review. Road Safety Research Report 19, Department for the Environment, Transport and the Regions, London UK 200I.

5. Duperrex O, Roberts I, Bunn F: Safety education of pedestrians for injury prevention. Cochrane Database Syst Rev 2002:CD001531.

6. MacGregor C, Smiley A, Dunk W: Identifying gaps in Child Pedestrian Safety: Comparing what children do with what parents teach. Transportation Research Record 1999, 1674:0724.

7. Malek M, Guyer B, Lescohier I: The epidemiology and prevention of child pedestrian injury. Accid Anal and Prev 1990, 22:20I-3।3.

8. Bunn F, Collier T, Frost C, Ker K, Roberts I, Wentz R: Traffic calming for the prevention of road traffic injuries: systematic review and meta-analysis. Injury Prevention 2003, 9:200-204.

9. Roberts I: Injuries to Child Pedestrians. Brit Med J 1995, 3 10:4|3-4|4.

10. Grayling T, Hallam K, Graham D, Anderson R, Glaister S: Streets Ahead: safe and liveable streets for children. Institute for Public Policy Research, London, UK 2000. 
11. Noble M, Penhale B, Smith G, Wright G, Dibden C, Owen T, Lloyd M: Indices of Deprivation. Department for the Environment, Transport and the Regions, London, UK 2000.

12. Posner J, Liao E, Winston F, Cnaan A, Shaw K, Durbin D: Exposure to traffic amongst urban children injured as pedestrians. Injury Prevention 2002, 8:23I-235.

13. Robinson WS: Ecological correlation and the behavior of individuals. American Sociological Review 1950, 15:35I-7.

14. Lancaster G, Green M: Deprivation, ill-health and the ecological fallacy. JR Statist Soc A 2002, 165:263-278.

15. Christie N: Social, economic and environmental factors in child pedestrian accidents: A research review. TRL Project Report I 16, Transport Research Laboratory, Crowthorne, Sussex 1995.

16. Bly P, Dix M, Stephenson C: Comparative Study of European Child Pedestrian Exposure and Accidents. Departments of the Environment, Transport and the Regions, London, UK 1999.

17. Anonymous: Guidance on Urban and Rural Area Definitions. [http://www.statistics.gov.uk/geography/urban rural.asp].

18. Anonymous: Casualties by road user type, child and adult pedestrianss, severity, county and unitary authority (UA) 2002. Road Casualties Great Britain: 2002: Annual Report 2003, Table 46a: II5-II7.

19. Denham C, White I: Differences in urban and rural Britain. Population Trends 1998, 91:23-34.

20. Wilding P: The $\mathbf{2 0 0 2}$ Quality Review of Road Accident Statistics. Road Accidents Great Britain: 200I: The Casualty Report 2002:32-27.

21. Bull J, Roberts B: Road accident statistics - a comparison of police and hospital information. Accid Anal and Prev 1973, 5:45-53.

22. James H: Under-reporting of road traffic accidents. Traffic Engineering and Control 1991, 32:574-583.

23. Cryer P, Westrup S, Cook A, Ashwell V, Bridger P, Clarke C: Investigation of bias after data linkage of hospital admissions data to police road crash reports. Injury Prevention 200I, 7:234-24I.

24. Keigan M, Broughton J, Tunbridge RJ: Linkage of Stats 19 and Scottish Hospital in-patient data - analysis for 1980 - 1985. Report TRL 420. Transport Research Laboratory, Crowthorne, Surrey 1999.

25. Plowden S, Hillman M: Danger on the Road; The Needless Scourge. Policy Studies Institute, London, UK 1984.

26. Abdalla IM: Statistical investigation and modelling of the relationships between road accidents and social characteristics. Phd Thesis, Napier University, Edinburgh, Scotland UK 1997.

27. Wakefield JC, Best NG, Waller L: Bayesian approaches to disease mapping. In Spatial Epidemiology, Methods and Applications Edited by: Elliot P, Wakefield J, Best N, Briggs D. Oxford University Press; 2000:104-127.

28. Bhattacharya S: A Result on Accident Proneness. Biometrika 1967, 54:324-325.

29. MacNab YC: A bayesain hierarchical model for accident and injury surveillance. Accid Anal and Prev 2003, 35:91-102.

30. Vistisen $D:$ A consistent method for estimating the effect of hot spot safety work. Traffic Engineering and Control 2002, 43:96-100.

31. Tunaru R: Hierarchical Bayesian models for road accident data. Traffic Engineering and Control 1999, 40:318-324.

32. Ihaka R, Gentleman R: A Language for Data Analysis and Graphics. Journal of Computational and Graphical Statistics 1996, 5:299-3।4.

33. The R Foundation for Statistical Computing: The R Environment. [http://www.r-project.org].

34. Venables WN, Ripley BD: Modern Applied Statistics with S. Springer Fourth2002.

35. Beale NR, Taylor G], Straker-Cook DMK: Is council tax valuation band a predictor of mortality. BMC Public Health 2002, 2: 17.

36. Stevenson M, Jamrozik K, Burton P: A case-control study of child pedestrian injuries in Perth, Western Australia. J Epidemiol Commun Health 1997, 50:280-287.

\section{Pre-publication history}

The pre-publication history for this paper can be accessed here:

http://www.biomedcentral.com/1471-2458/4/15/prepub
Publish with Bio Med Central and every scientist can read your work free of charge

"BioMed Central will be the most significant development for disseminating the results of biomedical research in our lifetime. "

Sir Paul Nurse, Cancer Research UK

Your research papers will be:

- available free of charge to the entire biomedical community

- peer reviewed and published immediately upon acceptance

- cited in PubMed and archived on PubMed Central

- yours - you keep the copyright
Biomedcentral 